\title{
Perspectives théoriques à l'égard des fonctions exécutives en contexte éducatif chez les enfants d'âge préscolaire
}

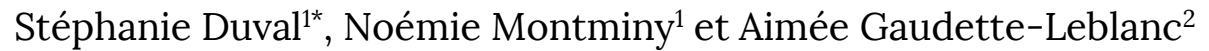

\section{RÉSUMÉ}

Cet article théorique vise à documenter l'évolution des perspectives qui ont été dégagées sur le développement des fonctions exécutives (FE) chez les enfants âgés entre 3 et 6 ans. Malgré la présence d'un accord général sur la définition plus large du concept de FE, un débat persiste toujours sur les composantes qui y sont associées, surtout chez les jeunes enfants. En effet, différentes propositions théoriques quant à la manière de conceptualiser les FE ont été proposées depuis le début des années 2000. Il est donc difficile de s'entendre sur les habiletés qu'il est possible d'observer chez les enfants d'âge préscolaire, notamment en contexte éducatif. Dans un tel contexte, il s'avère essentiel de miser sur l'observation des habiletés associées aux FE dans des situations quotidiennes et naturelles (p. ex. temps de jeu), ce qui permet ensuite à l'adulte d'accompagner l'enfant dans le déploiement de ces dernières. Prenant appui sur les perspectives théoriques dégagées dans cet article, notamment celle de Miyake et al. (2000), cet article propose des pistes d'observation et d'intervention permettant de soutenir les habiletés liées aux FE à l'éducation préscolaire, de manière à favoriser le développement et l'apprentissage de l'enfant, par extension sa réussite éducative.

\footnotetext{
${ }^{1}$ Université Laval, Faculté des sciences de l'éducation, Département d'études sur l'enseignement et l'apprentissage, Québec, Canada

${ }^{2}$ Université Laval, Faculté de musique, Programme d'éducation musicale, Québec, Canada

* Correspondance avec l'auteure : stephanie.duval@fse.ulaval.ca
}

Pour citer cet article : Duval, S., Montminy, N. et Gaudette-Leblanc, A. (2018). Perspectives théoriques à l'égard des fonctions exécutives en contexte éducatif chez les enfants d'âge préscolaire. Neuroéducation, 5(2), 93-108.

DOI: $\underline{\text { https://doi.org/10.24046/neuroed.20180502.93 }}$

Reçu le $1^{\text {er }}$ novembre 2017. Révision reçue le 27 avril 2018.

Accepté le 10 mai 2018. Publié en ligne le 19 septembre 2018.

Neuroéducation, 5(2), 93-108

ISSN: 1929-1833

Tous droits réservés C 2018 - Association pour la recherche en neuroéducation 


\section{Introduction}

Jusqu'à tout récemment, l'étude des fonctions exécutives (FE) a principalement été le domaine des neurologues et des neuropsychologues (Anderson, Jacobs et Anderson, 2008; Denckla, 1996; Diamond, 2006; Holmes-Bernstein et Waber, 1990). Une attention accrue est présentement portée envers ces fonctions cognitives de haut niveau dans le monde de l'éducation (p. ex. Diamond, 2016). Pour cause, les FE permettent d'assurer le «contrôle cognitif » de l'enfant, car elles lui permettent de raisonner, de planifier, de concentrer son attention et d'inhiber les comportements jugés non appropriés dans le contexte où il se trouve (Chevalier, 2010). De cette manière, les FE lui permettent de contrôler intentionnellement sa pensée et ses actions dans l'atteinte d'un but précis (Chevalier, 2010; Miyake et al., 2000).

$\mathrm{Au}$ plan international, de plus en plus de chercheurs soulignent l'importance d'étudier les FE chez l'enfant, particulièrement à la période de 3 à 6 ans qui s'avère critique pour leur développement (Diamond, 2016). De manière plus précise, des travaux font ressortir des liens entre les FE, définies comme un ensemble de processus cognitifs interdépendants, et la réussite éducative de l'enfant, dès son entrée à la maternelle (p. ex. Engel de Abreu et al., 2014). Des études ont d'ailleurs montré que les enfants de la maternelle qui démontraient de meilleures $\mathrm{FE}$, telles que mesurées à l'aide de tests standardisés, s'engageaient plus efficacement dans les situations d'apprentissage offertes en première année du primaire (Blair et Raver, 2014; Williford et al., 2013).

Ces études montrent qu'un intérêt est porté envers l'étude des FE en contexte éducatif, notamment lors de la transition de l'éducation préscolaire vers l'enseignement primaire. Ce moment, qui marque le passage de l'enfant d'un milieu où l'apprentissage par le jeu est valorisé (éducation préscolaire) vers un environnement où l'enseignement plus formel de domaines disciplinaires (p. ex. mathématiques, lecture, etc.) est préconisé (Moreno, Shwayder et Friedman, 2016), amène l'enfant à déployer plusieurs habiletés associées aux FE.

Si des études ont examiné le lien entre les FE de l'enfant et sa performance dans les tâches scolaires, peu d'entre elles ont spécifiquement porté sur les habiletés associées aux FE en contexte d'éducation préscolaire. Ces habiletés sont pourtant sollicitées lors de situations naturelles et quotidiennes de classe, par exemple en situation de jeu (Moreno et al., 2016). De plus, bien qu'une attention soit actuellement portée envers la réussite éducative de l'enfant dès son entrée à l'école (Bierman et Torres, 2016; Simard et al., 2013), on se questionne toujours sur les pratiques enseignantes les plus optimales à mettre en place en classe, de manière à favoriser le développement et l'apprentissage de chacun.

À l'avis de plusieurs chercheurs, on se questionne davantage sur comment intervenir auprès de l'enfant afin de l'amener à réaliser des apprentissages, souvent axés sur les domaines disciplinaires (p. ex. mathématiques) (p. ex. Hirsh-Pasek et al., 2008; Pellegrini, 2009). Cependant, dans une perspective développementale, soit celle privilégiée par les programmes éducatifs pour les enfants d'âge préscolaire (MEQ, 2006; MFA, 2007), on devrait miser sur comment les enfants apprennent, de manière à guider les pratiques enseignantes selon les besoins de chacun.

Dans cette perspective, l'enfant est perçu comme un apprenant actif qui, grâce à ses interactions avec le monde qui l'entoure, construit des connaissances, habiletés et attitudes qui lui permettent d'évoluer et de s'adapter continuellement à travers ses nouvelles expériences (HirshPasek et al., 2008). Afin de miser sur une approche centrée sur l'enfant et préconisant l'apprentissage actif (p. ex. situations de jeu), il semble primordial de comprendre l'évolution du développement des FE chez l'enfant d'âge préscolaire, de manière à examiner comment les habiletés ${ }^{1} \mathrm{y}$ étant reliées peuvent être mises à contribution (observation), pour ultimement mieux accompagner l'enfant (intervention).

Selon Moreno et al. (2016), en dépit d'un accord général sur la définition plus large du concept de $\mathrm{FE}$, un débat persiste toujours sur les composantes ${ }^{1}$ qui y sont associées, surtout chez les jeunes enfants. Il est donc difficile de s'entendre sur les habiletés qu'il est possible d'observer en contexte éducatif, afin de soutenir efficacement l'enfant dès l'éducation préscolaire. Quelles sont les composantes qui sont associées aux FE chez les enfants âgés entre 3 et 6 ans? Comment l'adulte peut-il observer les habiletés liées aux FE en contexte éducatif, pour ensuite ajuster ses interventions visant à les favoriser chez l'enfant? Voilà les questions qui sous-tendent cet article de nature théorique.

\section{Les FE chez l'enfant d'âge préscolaire}

Malgré la présence d'un débat permanent sur la nature des composantes liées aux FE à la petite enfance (c.-à-d., une composante globale ou des composantes différenciées) (Skogan et al., 2015; van der Ven et al., 2012), l'importance d'examiner leur développement à l'âge préscolaire est reconnue (Moreno et al., 2016). En effet, certaines périodes de la vie semblent plus propices au développement des FE (périodes dites critiques) (Kagan et Baird, 2004; Zelazo, 2013), ce qui justifie la pertinence d'y porter attention à ce moment charnière dans la vie de l'enfant.

En neuropsychologie, le concept de FE renvoie couramment à un terme «parapluie » qui englobe un ensemble de processus cognitifs nécessaires à la réalisation d'un comportement dirigé vers un but, sous la gouverne principale du cortex préfrontal et de ses réseaux (Bear, Connors et Paradiso, 2016; Roy, 2012). En éducation, les données de

\footnotetext{
${ }^{1}$ Le terme «habileté » est privilégié dans cet article, lequel réfère aux manifestations observables des FE à l'éducation préscolaire. Le terme « composante » fait plutôt référence à l'activité mentale pouvant être comprise dans ce que l'on appelle «la cognition » (Fortin et Rousseau, 2012). Dans cet article, la mémoire de travail, l'inhibition et la flexibilité mentale ont été retenues comme les composantes principales des FE.
} 
certaines études (Foy et Mann, 2012; Mann et al., 2016) mènent à penser que les FE pourraient constituer l'élément qui unit toutes les dimensions du développement global de l'enfant d'âge préscolaire ${ }^{2}$. Ainsi, on peut penser que les FE constitueraient la pierre angulaire du développement et de l'apprentissage de l'enfant et qu'autour d'elles convergeraient plusieurs stratégies, habiletés et connaissances (Duval et Bouchard, 2013).

Selon Fuhs, Farran et Nesbitt (2013), bien qu'il demeure pertinent de parler d'une seule FE plutôt que des FE (composante globale versus composantes différenciées) à l'âge préscolaire, force est de constater qu'une coordination de certaines habiletés associées aux FE s'avère primordiale dès l'entrée à l'école. Prenons l'exemple d'une situation de jeu incluant des blocs de bois : l'enfant pourrait alors user de ses habiletés d'inhibition en déplaçant minutieusement les blocs pour arriver à construire une tour formée de plusieurs étages. Il pourrait également mettre en œuvre sa flexibilité mentale, par exemple si un autre enfant propose une stratégie qui n'était pas envisagée initialement et qui contribue à bonifier la construction en cours. Lorsque l'enfant s'engage dans des situations qui lui permettent de coordonner ses habiletés liées aux FE, il arrive ainsi à mettre en place des actions et des pensées orientées vers des buts précis. Cet exemple illustre que plusieurs FE peuvent être sollicitées lors d'une même situation à l'éducation préscolaire.

De manière à bien les observer en contexte éducatif, il importe d'abord de bien comprendre ces composantes, d'autant plus qu'il est démontré que les FE de l'enfant de la maternelle prédisent ses succès futurs (Foy et Mann, 2012; Mann et al., 2016). À titre illustratif, Blair et Razza (2007) ont montré que les composantes «mémoire de travail» et « inhibition » permettraient de prédire le rendement scolaire des enfants en mathématiques et en lecture, dès leur entrée au primaire. Dans le même sens, une étude menée par Kroesbergen, Van Luit, Van Lieshout, Van Loosbroek et Van de Rijt (2009) a montré que chez les enfants de 5-6 ans, les FE «mémoire de travail» et «inhibition » étaient notamment associées aux compétences en mathématiques. Par ailleurs, des études ont aussi montré l'importance des FE dans le développement social des jeunes enfants, lequel s'avère également impliqué dans leur réussite éducative présente et ultérieure (Bull, Espy et Wiebe, 2008; Clark, Pritchard et Woodward, 2010; Röthlisberger et al., 2011).

Bien que ces travaux dévoilent des liens précis entre les composantes liées aux FE chez les enfants et leur réussite éducative, il importe de noter que l'émergence des connaissances associées au développement des FE chez l'enfant est encore récente (Roy, 2012). En effet, la transposition de connaissances acquises sur le développement des FE découle d'abord des travaux en neuropsychologie réalisés chez l'adulte. Les études menées auprès de l'enfant utilisent parfois la tendance générale en employant des tests conçus pour l'adulte (Ibid.), lesquels sont généralement effectués en laboratoire. Ainsi, s'il est vrai que l'acquisition des connaissances sur les FE issues des travaux en neuropsychologie chez l'adulte a permis de constituer un cadre de référence théorique sur leur développement des FE, il s'avère essentiel de demeurer prudent sur la transposition qu'on peut $y$ faire chez l'enfant. Effectivement, les propositions théoriques concernant la manière de conceptualiser les FE chez les enfants, basées sur ce que l'on connait du cerveau adulte, s'avèrent hypothétiques en raison du développement cérébral qui n'est pas à sa pleine maturité.

Le fait de s'intéresser à l'évolution développementale des FE chez l'enfant d'âge préscolaire pourrait permettre de dégager des pistes d'observation et d'intervention pour les adultes qui les accompagnent (p. ex. enseignants de maternelle), tel qu'il est proposé dans la discussion de cet article. À l'éducation préscolaire, l'observation quotidienne des enfants constitue un outil de premier ordre pour connaitre les potentionnalités de chacun afin de les accompagner dans leurs apprentissages (MFA, 2007). En observant les habiletés associées aux FE chez l'enfant, l'adulte pourra ainsi examiner ce que l'enfant est capable de faire seul par l'exploration de son environnement, et il pourra complexifier les situations d'apprentissage et de développement afin que ce dernier progresse harmonieusement, de manière à respecter son rythme qui lui est propre.

Pour y parvenir, il importe d'abord de dégager les perspectives théoriques actuelles qui sont proposées afin de comprendre le développement des FE chez les enfants. Peuton supposer que plusieurs habiletés composent les FE chez les enfants, tel que chez les adultes?

\section{Cadre théorique}

Les FE s'avèrent des fonctions psychologiques supérieures, qualifiées de complexes et étant influencées par l'environnement culturel et social (Luria, 1966). Certaines périodes de la vie (p. ex. 3 à 6 ans) sont plus critiques pour leur formation, car elles coïncident avec le développement du cortex préfrontal (De Luca et Leventer, 2008). Le développement de cette région cérébrale est caractérisé par une maturité ontogénétique particulièrement tardive (Dennis, 2006); les fonctions dévolues au cortex préfrontal du cerveau se développent ainsi graduellement pendant l'enfance, et ce, jusqu'à l'âge adulte. Bien que le cortex préfrontal n'atteigne pas sa maturité avant l'âge adulte, les éléments-clés qui le composent, tels que les FE, sont présents tôt dans la vie et doivent être reconfigurés tout au long de l'enfance (Roy, 2012). Ainsi, le développement des FE chez l'enfant est à la fois précoce et prolongé, en raison de la maturation du cortex préfrontal.

Selon Luria (1966), premier chercheur à avoir introduit le terme « FE » dans ses recherches, les fonctions cognitives de haut niveau se développent d'abord grâce aux interactions entre l'enfant et les adultes qui l'entourent (p. ex. parent,

\footnotetext{
${ }^{2}$ Le développement global peut être défini comme le développement simultané, intégré, graduel et continu de toutes les dimensions qui composent

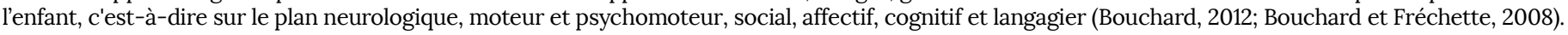


enseignant). Luria considère l'activité humaine selon une approche systémique, c'est-à-dire une approche permettant de considérer différents facteurs d'influence dans le développement et l'apprentissage de l'enfant. De ce point de vue, l'histoire personnelle de l'enfant exerce une influence sur son esprit, ce qui lui permet d'adapter ses comportements aux différents contextes dans lesquels il évolue (p. ex. contexte éducatif). Selon Luria (1966), Vygotski serait le premier chercheur à avoir examiné les principes qui soustendent la relation entre le cerveau et le comportement.

Selon la théorie vygotskienne, le développement de l'enfant est le fruit d'une genèse sociale et il doit être compris dans une perspective historico-culturelle. Vygotski (1997) affirme que le contexte social façonne les processus mentaux de l'enfant, en plus de faire partie intégrante de son processus développemental (Bodrova et Leong, 2010). De cette manière, les comportements individuels découlent de l'interaction entre l'enfant et l'environnement dans lequel il évolue. Vygotski $^{3}$ (1978) distingue deux catégories de processus mentaux : 1) les fonctions psychiques élémentaires, qui sont biologiquement déterminées et 2) les fonctions psychiques supérieures, qui ont des origines socioculturelles. La culture touche à la fois au développement des fonctions psychiques supérieures et à la façon dont elles sont acquises (Bodrova et Leong, 2010), tandis que les processus élémentaires produisent l'activité générale et fondamentale de la plupart des hémisphères cérébraux (Vocate, 1987). En somme, tant pour Vygotski que pour Luria, les FE transcendent l'expérience de l'enfant et reflètent le niveau culturel de son environnement social.

Suite aux travaux de Vygotski et de Luria, de nombreux chercheurs ont tenté d'étudier plus finement le développement des FE (p. ex. Diamond, 2013; Miyake et Friedman, 2012). Dans de nombreuses études portant sur les FE chez les enfants d'âge préscolaire, les chercheurs s'entendent sur le fait qu'il existerait au moins trois composantes fondamentales y étant liées : la mémoire de travail, l'inhibition et la flexibilité mentale (Jacob et Parkinson, 2015; Miyake et al., 2000).

\subsection{La mémoire de travail}

La mémoire de travail représente une pièce maîtresse dans le développement des FE. En contexte éducatif, elle permet à l'enfant de retenir une information et de l'utiliser d'une manière appropriée dans un autre contexte (Diamond, 2013). Cette composante des FE réfère ainsi à la capacité de stocker temporairement des informations en mémoire, afin de les manipuler et de les contrôler ultérieurement (Dekker, Ziermans et Swaab, 2016).

À l'éducation préscolaire, l'enfant pourrait par exemple user d'habiletés liées à la mémoire de travail lors de la lecture d'une histoire, lorsque l'adulte lui pose des questions sur les évènements et les personnages (p. ex. Dans cette histoire, quelle est l'activité préférée de l'ours? Que s'est-il passé avant qu'il parte en camping?). De même, si l'adulte invite l'enfant à faire des liens entre l'histoire et sa vie personnelle, ce dernier sera amené à utiliser différentes habiletés de mémoire (p. ex. Et toi, es-tu déjà allé en camping? Que faisais-tu comme activité? Est-ce semblable à ce que l'ours fait dans cette histoire?). Selon plusieurs auteurs (p. ex. Best et Miller, 2010; Best, Miller et Jones, 2009), cette FE s'améliore progressivement au cours de l'enfance et de l'adolescence, et ce, de façon linéaire.

\subsection{L'inhibition}

L'inhibition permet à l'enfant de contrôler des actions ou de résister à des interférences qui proviennent de stimuli externes et qui ne sont pas pertinents dans la situation actuelle. Cette composante est considérée comme une habileté préalable pour l'acquisition des autres FE (Dekker et al., 2016). À l'éducation préscolaire, l'enfant pourrait user d'habiletés liées à l'inhibition lors d'un jeu de construction, par exemple. En voulant construire une tour de huit étages (comme celle devant sa maison), un enfant pourrait alors être amené à contrôler ses gestes pour que la tour ne s'effondre pas en cours de construction. Cet enfant devra alors adapter ses gestes en fonction de l'environnement, de manière à répondre à l'objectif qu'il s'est lui-même fixé. Si la tour s'effondre, il sera amené à réguler ses émotions (p. ex. se féliciter d'avoir fait cinq étages au lieu d'être fâché de ne pas en avoir réalisé huit), ce qui est également associé à l'inhibition.

À l'âge préscolaire, les capacités d'inhibition s'améliorent rapidement. C'est plus précisément vers l'âge de 4 ans que l'enfant présente des habiletés de base liées à l'inhibition. À cet âge, l'enfant réussirait mieux les tâches d'inhibition motrices que celles d'inhibition verbale (Best et Miller, 2010). Cette composante des FE poursuivrait son développement de façon graduelle et linéaire entre l'âge de 5 à 8 ans. (Best et al., 2009).

\subsection{La flexibilité mentale}

Cette composante réfère à la capacité de l'enfant à changer de tâche ou de stratégie afin de passer d'une opération cognitive à une autre (Chevalier, 2010). De manière plus précise, cette FE lui permet de désengager son attention visà-vis une situation, afin de s'engager dans une nouvelle, en fonction des exigences de cette dernière. À l'éducation préscolaire, l'enfant pourrait user d'habiletés liées à la flexibilité mentale en suivant les règles associées à une routine précise (p. ex. transiter du coin blocs vers la collation) ou en s'engageant dans un rôle au coin jeu symbolique (p. ex. passer du statut «enfant » à celui de « commis d'épicerie », en suivant les règles associées au jeu).

Rappelons qu'actuellement, un vif intérêt est porté envers l'étude des FE en contexte éducatif, notamment à l'éducation

${ }^{3}$ S'écrit Vygotsky, dans les références, dans le cas de ses publications en anglais. 
préscolaire (p. ex. Moreno et al., 2016). Bien que plusieurs chercheurs s'entendent sur le fait que la période préscolaire constitue un moment où l'on observe d'importants changements cognitifs (Diamond, 2013; Hoskyn, Iarocci et Young, 2017), très peu arrivent à un consensus quant à la forme que prennent ces derniers. Les prochaines sections visent à dégager les principales manières de conceptualiser les FE chez l'enfant âgé entre 3 et 6 ans, à partir des études qui ont été diffusées depuis le début des années 2000.

\section{Différentes perspectives développementales}

Knapp et Morton (2017) soulignent que plusieurs transformations particulièrement prononcées apparaissent dans le développement des FE lors de la petite enfance. Selon les chercheurs, la maturation du cerveau suit un continuum de développement prolongé, en concordance avec le développement du cortex préfrontal qui s'échelonne sur une longue période de temps (Ibid.). En effet, les circuits neuronaux associés au développement des FE s'avèrent lents à se développer, car les régions préfrontales du cerveau atteignent leur maturité seulement au début de l'âge adulte (Lenroot et Giedd, 2006). De manière similaire, les habiletés liées aux FE continuent de s'améliorer dans la deuxième décennie de la vie. L'existence de ces similitudes dans les trajectoires de développement entre le cortex préfrontal du cerveau et les habiletés liées aux $\mathrm{FE}$ a conduit à beaucoup de recherches dédiées à examiner cette relation plus en profondeur.

Des études ont examiné les différences liées à l'âge dans le fonctionnement du cerveau au cours de la réalisation de tâches liées aux FE, et ce, pour suggérer que ces deux processus de développement semblent étroitement liés. Cependant, il existe un certain désaccord quant à la façon de décrire l'évolution du cortex préfrontal tout au long de l'enfance et d'expliquer l'effet de celle-ci sur le développement des FE (Knapp et Morton, 2017).

Il existe également un certain désaccord sur la manière de conceptualiser les FE chez l'enfant. L'une des grandes approches permettant de conceptualiser les FE pointe vers une structure unitaire, qui suggère qu'une seule FE serait observée chez l'enfant, laquelle serait composée de plusieurs habiletés communes et indissociables.

\subsection{Modèle théorique à une composante (structure unitaire)}

Un nombre important d'études concernant le développement des FE chez les enfants âgés de 3 ans et moins (p. ex. Wiebe et al., 2011; Willoughby et al., 2010; Willoughby, Wirth et Blair, 2012) présentent un modèle de FE à structure unitaire, lequel diffère de la structure proposée pour les enfants plus âgés.

L'hypothèse selon laquelle le fonctionnement exécutif serait d'abord composé d'un construit unique (une seule FE) correspond à la théorie selon laquelle les activités mentales seraient initialement générales, puis se spécialiseraient progressivement avec l'âge et l'expérience (Clark et al., 2013). Selon cette perspective théorique, les composantes associées aux FE (p. ex. mémoire de travail, inhibition) seraient relativement indifférenciées, notamment en début de vie (Diamond, 2013; Hughes et al., 2009; Roebers, 2017; Wiebe et al., 2011).

Bien que certaines études supportent également une structure unitaire dans le développement des FE chez les enfants âgés entre quatre et cinq ans (p. ex. Fuhs et Day, 2011; Shing et al., 2010; Wiebe, Espy et Charak, 2008), il semble qu'on pourrait différencier les composantes associées aux FE au fur et à mesure que l'enfant grandit, et ce, à partir de l'âge de 4 ans environ. En fait, il en reste encore beaucoup à apprendre sur le moment où la transition de la structure unitaire vers la structure nommée « unité/diversité 》 émerge, telle que suggérée par Miyake et Friedman (2012).

\subsection{Le modèle théorique unité/diversité}

Les analyses factorielles confirmatoires effectuées dans les travaux de Miyake et al. (2000) ont montré que les FE peuvent être conceptualisées en trois composantes principales : la mémoire de travail, l'inhibition et la flexibilité mentale. Ces trois composantes associées aux FE peuvent être conceptualisées dans un cadre nommé « unité/diversité » (Fujisawa, Todo et Ando, 2016). Cette manière de les conceptualiser suppose que ces trois composantes sont corrélées les unes aux autres et partagent plusieurs processus sous-jacents communs (unité), mais qu'elles présentent également certains degrés de séparabilité (diversité) (Miyake et al., 2000).

Suite aux travaux de Miyake et al. (2000), plusieurs chercheurs ont proposé des modèles théoriques suggérant une structure à trois composantes dans l'étude des FE chez les enfants d'âge préscolaire (p. ex. Best et Miller 2010; Best et al., 2009; Garon, Bryson et Smith, 2008). Selon ces auteurs, les composantes des FE partageraient des caractéristiques communes, mais elles seraient également indépendantes. Par exemple, Garon et al. (2008) ont effectué des analyses factorielles confirmatoires dévoilant que trois composantes formaient le concept de FE (mémoire de travail, inhibition, flexibilité mentale). Lors de leurs analyses, les chercheurs ont démontré que ces composantes s'avéreraient partiellement indépendantes, tout en étant corrélées entre elles.

Toujours en s'appuyant sur les travaux de Miyake et al. (2000), Best et Miller (2010) ont défini un modèle à trois composantes ayant différentes trajectoires développementales. Plus précisément, ces derniers ont proposé que le degré de corrélation et d'indépendance des $\mathrm{FE}$ variait selon l'âge de l'enfant. Par exemple, les chercheurs soulignent que l'inhibition se développerait plus rapidement pendant les années préscolaires (3-6 ans), tandis que la mémoire de travail et la flexibilité mentale évolueraient de manière linéaire et progressive tout au long de l'enfance.

En 2012, Miyake et Friedman ont proposé que chacune de ces composantes pouvait être décomposée (diversité), tout en conservant des particularités communes (unité). La figure 1 illustre le modèle de Miyake et Friedman (2012), par une représentation schématique de l'unité et de la diversité. Cette figure montre que les habiletés reliées à chacune des 
composantes (p. ex. mémoire de travail) comportent des processus cognitifs communs aux trois FE (FE commune). Toutefois, chaque composante est constituée de processus cognitifs spécifiques (p. ex. processus spécifique à la mémoire de travail) (voir figure 1).

La figure 1 dévoile également qu'en comparaison avec les processus spécifiques à la mémoire de travail et la flexibilité mentale, l'inhibition s'avère uniquement comprise dans la FE commune (unité). En effet, cette composante (inhibition) serait la seule à s'expliquer par l'unité, car elle présente des particularités communes aux autres composantes (Miyake et Friedman, 2012). Plus précisément, Miyake et Friedman (2012) ont montré que l'inhibition corrèle fortement et significativement avec les deux autres composantes, ce qui mène à la considérer comme une « FE commune » (unité).

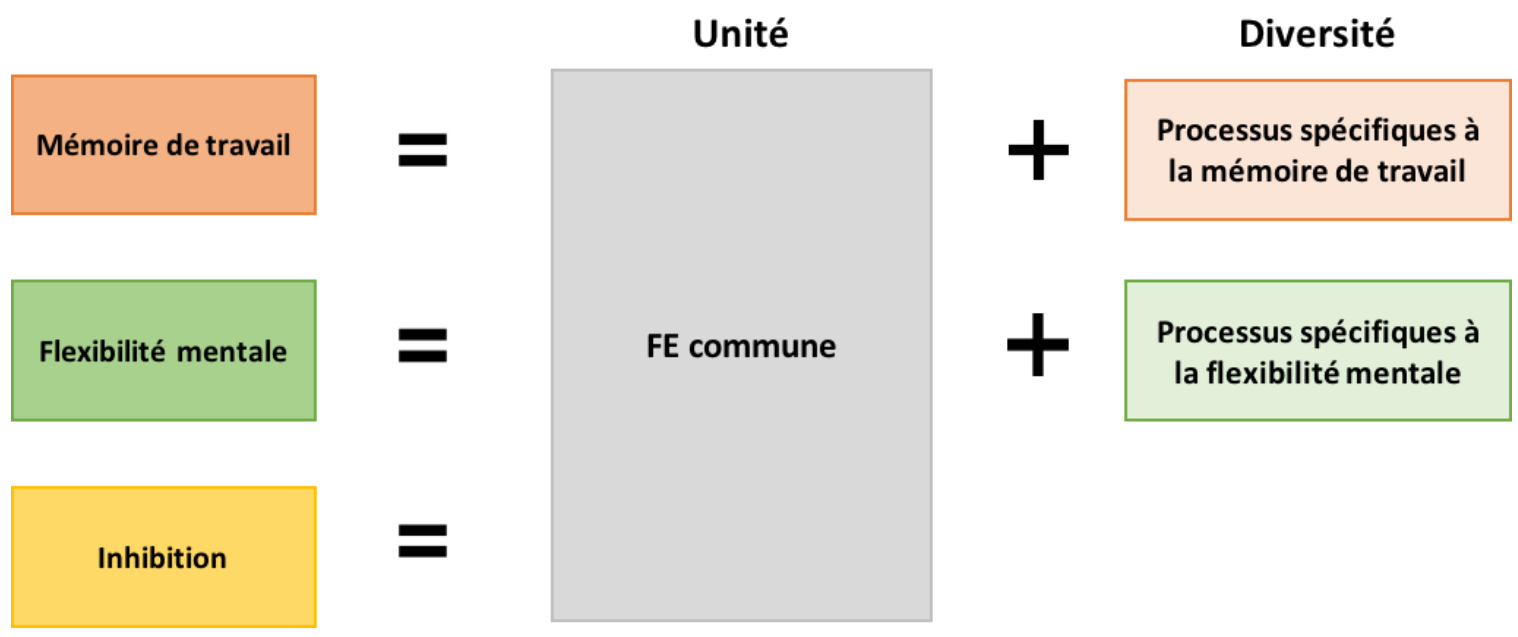

Figure 1. Représentation schématique de l'unité et de la diversité des trois composantes principales des FE. (figure inspirée de Miyake et Friedman, 2012).

Ce qui émerge de la proposition théorique dégagée par ces chercheuses pointe vers la complexité de la composante « inhibition ». Celle-ci représente une composante en soi, mais elle constitue également un facteur général de contrôle exécutif qui influence les autres composantes y étant liées (Ibid.). Suite aux travaux de Miyake et Friedman (2012), Diamond (2013) a suggéré qu'une relation bidirectionnelle existait entre l'inhibition et la mémoire de travail, les décrivant comme des composantes séparées, mais fortement reliées.

Il importe de mentionner que les modèles proposés par Miyake et ses collaborateurs (Miyake et al., 2000; Miyake et Friedman, 2012) ont été élaborés à partir d'un échantillon de participants adultes. Cependant, il s'avère pertinent de les présenter, car plusieurs auteurs se sont par la suite inspirés de ces modèles en tentant de les appliquer à des échantillons d'âges variables. Il est à souligner que le développement des FE apparait selon un modèle de stades développementaux plutôt que de manière statique. Ainsi, les FE de l'enfant progressent selon le rythme de développement de chacun, et non selon un cadre fixe et invariable pour tous les individus. Il s'avère donc difficile de préciser l'âge exact où l'on peut considérer l'un ou l'autre des modèles théoriques.

À titre illustratif, de nombreuses études ont montré qu'une diversité de FE existait chez les enfants d'âge primaire (7 à 12 ans) (p. ex. Engelhardt et al., 2015; Huizinga, Dolan et van der
Molen, 2006; Lehto et al., 2003; Shing et al., 2010), tandis que d'autres chercheurs ont démontré que cette différenciation pouvait se produire un peu plus tôt (p. ex. Miller et al., 2012; Usai et al., 2013), tel qu'à l'âge préscolaire (3 à 6 ans). Néanmoins, un aspect commun unit ces travaux: ils proposent un modèle théorique formé de trois composantes (mémoire de travail, flexibilité mentale et inhibition), lesquelles s'avèrent corrélées entre elles, tout en présentant un caractère dissociable. Outre ces travaux, d'autres chercheurs identifient une structure des FE à deux composantes.

\subsection{Modèle théorique à deux composantes}

Des chercheurs suggèrent une structure formée de deux composantes chez les enfants âgés entre 4 et 5 ans (Lee, Bull et Ho, 2013; van der Ven et al., 2012). Cette structure serait plus précisément composée de 1) l'inhibition, puis de 2) la mémoire de travail et de la flexibilité mentale en tant que FE combinées (Lee et al., 2013; van der Ven et al., 2012). Par ailleurs, d'autres chercheurs (p. ex. Miller et al., 2012; Usai et al., 2013) ont montré l'existence d'un autre modèle théorique, lequel serait formé de ces deux composantes : 1) la mémoire de travail, puis 2) l'inhibition et la flexibilité mentale (FE combinées). En 2015, Monette, Bigras et Lafrenière (2015) ont supporté ce dernier modèle, en suggérant que les composantes « mémoire de travail » et « inhibition/flexibilité mentale » pouvaient être observées chez les enfants âgés 
entre 4 et 5 ans. Selon ces chercheurs, ces deux composantes se développeraient en premier durant l'enfance.

Quoi qu'il en soit, ces différentes études, voire ces différentes manières de conceptualiser les $\mathrm{FE}$, démontrent que leur structure tend à se transformer avec l'âge des enfants. C'est pourquoi Johansson, Marciszko, Brocki et Bohlin (2015) soutiennent qu'on peut observer des améliorations graduelles dans les composantes qui forment les $\mathrm{FE}$, lesquelles deviennent de plus en plus complexes avec l'âge. Un dernier modèle théorique s'oriente d'ailleurs vers une perspective hiérarchique et évolutive de la structure des FE.

\subsection{Perspective liée à un développement hiérarchique des FE}

Selon Johansson et al. (2015), les FE seraient d'abord composées par une structure unitaire (0-3 ans), avant d'être séparées en deux composantes vers l'âge de 3 à 4 ans. Un modèle formé de trois composantes serait ensuite dégagé, lesquelles apparaitraient plus tard dans le développement de l'enfant (Ibid.). Ainsi, il semble que les différentes perspectives théoriques qui ont préalablement été dévoilées puissent être réconciliées, en supposant que l'inhibition, la flexibilité mentale et la mémoire de travail constitueraient différentes composantes qui se différencieraient progressivement les unes des autres, et ce, en fonction de l'âge de l'enfant (Clark et al., 2013; Wiebe et al., 2011).

En ce sens, Garon et al. (2008), de même que Garon, Smith et Bryson (2013), ont proposé que les composantes associées aux FE se développeraient de manière séquentielle. Plus précisément, on verrait d'abord apparaitre la mémoire de travail en tant que composante distincte, suivie de l'inhibition, puis de la flexibilité mentale. Ainsi, on pourrait affirmer que ces trois composantes s'avèrent indépendantes, mais que le développement de chacune serait lié à celui des autres. Dans le même ordre d'idées, Diamond (2006) suggère que les FE représentent des processus cognitifs indépendants qui présentent une trajectoire développementale différente. Bien que la période située entre 3 et 6 ans s'avère critique pour leur développement, ce dernier s'avère progressif et linéaire, en plus d'être différent pour chaque enfant (rythme de développement unique) (Diamond, 2002).

\section{Synthèse des différentes perspectives théoriques dégagées}

Les sections précédentes ont permis de dégager une perspective évolutive dans le développement du fonctionnement exécutif durant l'enfance (3-6 ans). On peut notamment observer que la structure des FE se modifie progressivement au fil du développement, en raison de la maturation du cortex préfrontal (Knapp et Morton, 2017). En effet, différentes conceptions des FE ont été proposées depuis le début des années 2000, selon le point de vue théorique et le groupe d'âge examiné. Étant donné que la procédure d'évaluation et l'interprétation des résultats sont majoritairement basées sur l'un ou l'autre des modèles proposés pour examiner les habiletés liées aux FE chez l'enfant, il demeure important d'envisager différentes manières de conceptualiser les FE en cours de développement (Ibid.).

Les différentes conceptualisations des FE tendent historiquement à se situer selon deux grandes approches : 1) la perspective unitaire, qui met en évidence des composantes communes et indissociables des FE; et 2) la perspective présentant des composantes liées aux FE différenciées et interreliées (Knapp et Morton, 2017). En fait, il existe des preuves considérables qui soutiennent la perspective unitaire des FE chez le jeune enfant, y compris une forte corrélation entre les mesures utilisées (Carlson, Mandell et William, 2004; Friedman et Miyake, 2004). On verrait ensuite apparaitre une croissance progressive liée à la performance des FE au cours de la période comprise entre 4 et 6 ans. Des analyses factorielles confirmatoires ont également montré que plusieurs composantes seraient associées aux FE à partir de l'âge de quatre ans environ. Enfin, les changements liés à la structure des FE durant l'enfance seraient liés à la complexité hiérarchique des composantes (mémoire de travail, inhibition et flexibilité mentale) y étant associées, qui amènent les enfants à résoudre des problèmes de plus en plus difficiles au fur et à mesure qu'ils se développent (Zelazo et Müller, 2010).

Le développement des FE a traditionnellement été étudié à partir de perspectives biologiques ou neuropsychologiques en lien avec le développement du cortex préfrontal, qui devient mature plus lentement que d'autres régions du cerveau (Garon et al., 2008). Cependant, des recherches plus récentes ont mis l'accent sur les influences environnementales associées aux différences individuelles dans les FE et à son développement (Bernier et al., 2011; Raver, Blair et Willoughby, 2013; Rhoades et al., 2011). Ces études montrent notamment qu'il s'avère possible de soutenir les FE chez les enfants d'âge préscolaire, soit lors d'une étape transitoire importante dans leur développement (Fujisawa et al., 2016). Considérant les changements développementaux dans les FE lors de cette période, il serait plus facile de distinguer les composantes y étant liées au fur et à mesure que l'enfant se développe, soit à partir de 4 ans environ. On peut alors se poser la question suivante : à partir 4 ans, estce possible de distinguer les composantes associées aux FE chez l'enfant, à l'aide d'habiletés observées en contexte éducatif?

Selon Isquith, Gioia et Epsy (2004), il est d'abord nécessaire d'observer les habiletés associées aux FE quotidiennement en contexte éducatif pour ensuite les soutenir de manière efficace. Effectivement, l'observation est le premier jalon de lintervention éducative (MFA, 2007), car elle permet à l'enseignant de situer le niveau de développement global des enfants qui composent sa classe. Mieux connaitre et comprendre chacun des enfants, grâce aux observations effectuées lors de situations naturelles de classe, offre à l'adulte des pistes pour ensuite planifier et ajuster ses interventions, de manière à mieux soutenir les 
apprentissages et le développement de chacun. En ce sens, les prochains paragraphes ont pour objectif de discuter des habiletés observables liées aux FE en contexte éducatif, plus précisément en maternelle (4 et 5 ans).

\section{Discussion}

Selon Kaufman (2010), l'environnement joue un rôle majeur dans le développement des FE, notamment en raison de la plasticité considérable du cerveau lors de la période de la petite enfance, qui réfère à la malléabilité des structures cérébrales, lesquelles disposent d'une grande capacité d'adaptation aux demandes de l'environnement (OCDE, 2007). C'est pourquoi Bransford, Brown et Cocking (2000) soulignent que le développement de l'enfant n'est pas seulement 《préprogrammé » par les gènes. Effectivement, l'expérience, notamment les relations affectives, y contribue également de façon importante, en modifiant les structures du cerveau. Dans le même ordre d'idées, Hoskyn et al. (2017) soulignent que le passage du temps ne permettrait pas, à lui seul, d'assurer le développement des habiletés associées aux FE. Il y a donc tout lieu de miser sur la qualité et la richesse des expériences vécues chez l'enfant d'âge préscolaire afin d'optimiser le développement de ses FE (Diamond, 2016). Il s'avère aussi nécessaire d'observer efficacement les habiletés associées aux FE et d'adapter ses pratiques enseignantes en fonction du développement de celles-ci.

En contexte de classe, plusieurs situations naturelles et quotidiennes proposées par l'adulte (p. ex. arrivée du matin, temps de jeu) permettent à l'enseignant d'observer les habiletés associées aux FE chez les enfants, lesquelles sont associées aux dimensions de son développement global. Par exemple, lors d'une causerie en grand groupe, un enfant qui parvient à écouter et à participer aux échanges peut démontrer qu'il est en mesure de respecter le tour de parole, d'être attentif aux propos partagés, d'organiser ses idées et d'adapter son discours. Ces habiletés sont respectivement associées à l'inhibition, à la mémoire de travail et à la flexibilité mentale, lesquelles lui permettent de se développer globalement. En effet, ces habiletés permettent à l'enfant de s'investir dans la causerie en partageant ses idées et ses intérêts (développement affectif), d'interagir avec ses pairs (développement social), de participer aux échanges et exprimer son point de vue (développement langagier), tout en demeurant concentré sur la visée de la discussion. Cela l'amènera à réfléchir et à planifier ses réponses en lien avec le sujet discuté (développement cognitif). Par ailleurs, il peut être difficile, pour un enfant de cet âge, d'attendre son tour de parole. L'enfant peut comprendre qu'il n'est pas approprié, sur le plan social, de couper la parole de ses pairs. Toutefois, il n'arrive pas nécessairement à mettre en application ce qu'il a compris, c'est-à-dire qu'il peut être conscient qu'un comportement n'est pas souhaité, sans pour autant être en mesure de le retenir.

Somme toute, cet exemple démontre que l'observation des différentes habiletés associées aux FE en contexte de classe permettrait à l'enseignant de mieux connaitre le profil développemental de chacun des enfants. L'observation contribue ainsi à la qualité du soutien offert par l'adulte, car elle permet à l'enseignant d'adapter ses interventions en fonction des besoins et des potentionnalités de chacun, tel qu'il les aura observés (p. ex. De quoi l'enfant est-il capable? Quels sont ses défis? Quel matériel est plus opportun pour le soutenir?).

\subsection{Quelques pistes d'observation et d'intervention à l'éducation préscolaire}

La section qui suit présente des pistes d'observation et d'intervention en milieu éducatif (c.-à-d. en fonction de la routine quotidienne de classe), et ce, en tissant des liens avec les propositions théoriques dégagées précédemment. De manière plus précise, le modèle théorique proposé par Miyake (2012) a été retenu en vue d'organiser les idées discutées ci-dessous. Il est donc suggéré d'observer différentes habiletés reliées à la mémoire de travail, l'inhibition et la flexibilité mentale dans le cadre de différentes situations d'apprentissage et de développement offertes en classe d'éducation préscolaire. Plus précisément, les jeux de tables, de cartes et de règles, les jeux de coopération et les jeux symboliques semblent être des occasions où l'adulte peut observer et soutenir différentes habiletés liées aux FE. Mentionnons que les pistes proposées ci-dessous, bien que basées sur les propositions théoriques présentées précédemment, sont des suggestions faites par les auteures.

\subsubsection{Jeux de table, de cartes et de règles}

En contexte d'éducation préscolaire, les enfants ont souvent l'occasion de réaliser des jeux de table. Lors de ce type de jeu, l'adulte peut observer différentes habiletés associées aux FE. Effectivement, les enfants doivent habituellement rester dans une posture assise et éviter de porter attention aux distractions de l'environnement qui les entoure. Il leur faut ensuite mémoriser et manipuler des informations d'ordre visuelles et auditives. Prenons un jeu de Cherche et Trouve : l'enfant devra d'abord mémoriser les règles du jeu, pour ensuite concentrer son attention sur les caractéristiques de l'objet à trouver en vue de les conserver en mémoire. Il devra ensuite faire abstraction d'un ensemble plus ou moins grand de stimuli visuels et tenter de retrouver le symbole qu'il a en tête. Dans cette situation, l'inhibition et la mémoire de travail sont sollicitées. Dans le cas où le jeu de Cherche et Trouve présente plusieurs planches différentes, l'enfant recherchera alors le même symbole dans plusieurs environnements différents. Autrement dit, il devra s'adapter et repérer le même symbole à un endroit différent. Cela fera appel à sa flexibilité mentale.

De façon similaire, l'enfant à qui l'on offre de participer à un jeu de mémoire sera alors invité à faire abstraction des stimuli qui l'entourent, afin de maintenir son attention sur les stimuli visuels qui lui sont présentés. Il devra par exemple mémoriser l'emplacement d'une image en vue de la retrouver et de l'associer à une carte présentant la même image. Dans cette situation, l'inhibition et la mémoire de travail s'avèrent des 
composantes sollicitées par l'enfant. De plus, ses capacités liées à la flexibilité mentale peuvent être mises à contribution, en expérimentant différentes stratégies en vue de résoudre des problèmes rencontrés pendant le jeu.

D'autres types de situations, telles que la réalisation d'un casse-tête ou la participation à un jeu de construction, demandent aussi à l'enfant de manipuler des objets afin de reproduire une image. Par conséquent, ce dernier doit garder en mémoire la représentation de ce qu'il souhaite reproduire. De façon similaire, il est possible d'observer des habiletés associées à la mémoire de travail lors de la reproduction de séries ou de séquences. Par exemple, lorsqu'un enfant s'investit dans un jeu nécessitant de dupliquer une frise ou d'enfiler des billes selon un ordre déterminé, il doit maintenir en mémoire différentes informations d'ordre visuel, en plus de les manipuler afin de reproduire un ensemble.

Finalement, de nombreux jeux proposent à l'enfant de diviser ou de catégoriser des cartes selon une couleur ou un symbole précis. Certains de ces jeux comprennent une opération (p. ex. diviser des cartes selon leur couleur), d'autres en comprennent plusieurs (p. ex. diviser les cartes selon leur couleur et la forme qui est représentée). Entre les âges de 3 et 4 ans, il semble plus difficile pour l'enfant de persévérer si la tâche demandée implique deux opérations (Best et Miller, 2010). En effet, si deux consignes sont données de façon simultanée, cela implique que l'enfant soit en mesure de maintenir la seconde consigne en mémoire pendant qu'il applique la première.

Au fur et à mesure que l'enfant grandit, la capacité à retenir deux règles simultanément devient plus facile, notamment lorsqu'il est soutenu par l'adulte dans les situations de jeu. De surcroit, dans plusieurs jeux de table, une nouvelle règle s'avère graduellement présentée à l'enfant, ce qui modifie le cours de la partie (p. ex. passer son tour, piger une ou des cartes supplémentaires, changer l'ordre dans lequel les joueurs sont invités à jouer, etc.). En plus d'exiger de l'enfant qu'il mémorise la signification des symboles, des règles et des objectifs du jeu, ce dernier est alors invité à s'adapter (flexibilité mentale).

Enfin, les faits relevés dans les paragraphes précédents démontrent qu'il est possible d'observer plusieurs habiletés associées aux FE à travers des jeux de table, de cartes et de règles. Mis à part ces types de jeu, les situations impliquant davantage d'interactions sociales, telles que le jeu coopératif, permettent également à l'adulte d'observer des habiletés liées aux FE chez l'enfant.

\subsubsection{Jeux coopératifs}

Lors d'un jeu de coopération, l'enfant est invité à mettre en œuvre plusieurs habiletés liées aux FE. Lors de ses interactions avec ses pairs, il doit d'abord prêter une attention soutenue à l'autre, en plus d'apprendre à considérer d'autres points de vue, à négocier et à faire des compromis (Hoskyn et al., 2017). Lorsque l'enfant devient en mesure de prêter un bon niveau d'attention à l'autre, il arrive alors à identifier l'état émotionnel dans lequel celui-ci se présente à lui. Il peut ensuite adapter son comportement en conséquence (Carlson et Wang, 2007).

Lorsqu'il vit des conflits avec ses pairs, l'enfant doit être en mesure de considérer la perspective de l'autre (flexibilité mentale), tout en l'écoutant et en inhibant ses réponses. Le fait de tenter de trouver une solution qui répondra aux besoins et aux envies des deux parties amène l'enfant à réguler ses émotions, ce qui lui permet de coopérer avec les autres. Ainsi, les jeux qui permettent la coopération seraient associés à différentes tâches reliées à l'inhibition et à la flexibilité mentale (Carlson et Wang, 2007). Afin d'amener l'enfant à coopérer avec ses pairs, plusieurs chercheurs suggèrent de miser sur des jeux qui lui permettent de s'investir dans des interactions sociales régulières (p. ex. jeux symboliques), lesquelles permettent de développer ses habiletés cognitives, telles que les FE (Goble et Pianta, 2017; Vygotski, 1978).

\subsubsection{Jeu symbolique}

Le jeu symbolique peut être défini comme une situation d'apprentissage et de développement où l'enfant crée une situation imaginaire, joue un rôle et suit des règles (Vygotski, 1978). L'enfant prend plaisir à s'y investir, par exemple, en se déguisant et en interprétant des personnages qui s'avèrent symboliques pour lui (p. ex. jouer à la maman/au papa). Lors de ce type de jeu, l'enfant est amené à inhiber certains comportements spontanés et en adopter de nouveaux en vue de faire progresser le scénario de jeu prévu.

Par exemple, l'enfant qui revêt le rôle de patient dans un scénario de clinique médicale devra contrôler ses envies d'utiliser le stéthoscope (matériel réservé au médecin) pour conserver son rôle et enrichir le scénario. En faisant appel à des habiletés liées à la mémoire de travail, il pourra se représenter mentalement le scénario proposé et le rôle qui lui est attribué, en l'associant aux éléments de sa propre vie. Par exemple, un enfant qui joue le rôle de commis d'épicerie lors d'un jeu symbolique pourra se baser sur les expériences qu'il a vécues au marché au courant de l'été. Finalement, au cours du jeu symbolique, l'enfant peut mettre en place des habiletés liées à la flexibilité mentale, en s'ajustant aux changements proposés par ses pairs (p. ex. l'épicerie prend en feu) et en modifiant rapidement sa représentation de la situation. Le jeu symbolique exige donc le déploiement de nombreuses habiletés reliées aux FE.

Moreno et al. (2016) suggèrent que d'observer les habiletés liées aux FE lors du jeu symbolique, qui s'avère signifiant pour l'enfant, permet de le situer dans sa zone proximale de développement (ZPD), soit de favoriser son plein potentiel en respectant ses besoins et son propre rythme d'apprentissage (Vygotski, 1997). Le jeu devient ainsi un contexte par excellence pour favoriser les FE de l'enfant d'âge préscolaire, d'autant plus que le contexte de groupe dans lequel évolue l'enfant lui permet de prendre plaisir à partager son monde imaginaire. 
Tel que vu précédemment, Vygotski (1997) a soutenu que la culture s'avère un produit de l'activité sociale chez l'humain et, pour cette raison, l'étude du comportement de l'enfant nous conduit directement vers le volet social de son développement. Par conséquent, Fleer, Veresov et Walker (2017), suggèrent que les FE devraient être considérées dans un contexte social, tel que dans un jeu symbolique qui implique plusieurs enfants, car il crée les conditions optimales pour soutenir le développement de l'enfant. De même, s'appuyant sur les travaux de Vygotski, Bodrova, Germeroth et Leong (2013) soulignent la pertinence d'examiner les habiletés associées aux FE à travers le jeu symbolique, notamment lorsqu'il est mature. En effet, il ne suffit pas d'offrir des situations de jeu symbolique en classe ; encore faut-il observer et étayer le jeu des enfants afin qu'il se complexifie et qu'il amène des gains chez ces derniers.

5.2 Le jeu symbolique mature: contexte clé pour le développement des $\mathrm{FE}$

En parallèle aux travaux de Vygotski, Elkonin (2005) a introduit le concept de jeu mature, qui constitue une forme d'activité unique pour l'enfant, notamment lorsqu'il est soutenu par l'adulte. Le jeu symbolique mature constituerait la forme la plus optimale pour favoriser les FE de l'enfant (Bodrova et Leong, 2007; Elkonin, 2005), car il l'amène à inventer des scénarios signifiants et complexes qui se rapprochent de sa réalité (Diamond, 2013). C'est pourquoi Blair et Raver (2014) soulignent que le jeu symbolique mature, planifié de manière intentionnelle, sert de véhicule pour développer les FE chez les enfants, de même que le langage, le vocabulaire et la créativité.

Selon Kelly et Hammond (2011), l'inhibition serait la première FE à émerger pendant l'âge préscolaire (3-6 ans), soit au même moment où le jeu symbolique est également à son apogée. En ce sens, Elias et Berk (2002) ont mené une étude longitudinale qui a montré que lorsque l'enfant s'engageait dans un jeu symbolique de haut niveau avec ses pairs au courant de l'année scolaire, il démontrait davantage d'habiletés d'inhibition à la fin de l'année, tel que mesurées par l'observation en contexte naturel lors de périodes de rangement. De manière plus précise, les enfants qui mettaient en œuvre des habiletés associées aux FE dans le cadre de leurs jeux symboliques étaient ensuite en mesure de les transférer dans d'autres situations de classe (p. ex. lors du rangement des ateliers).

Outre la capacité d'inhibition, on voit apparaitre, dans le jeu symbolique mature, des habiletés reliées à la mémoire de travail et à la flexibilité mentale (Diamond, 2013). Effectivement, dans ce contexte de jeu, l'enfant est amené à assumer et à maintenir un rôle spécifique en s'engageant constamment dans des actions y étant liées, en prenant part à des interactions qui correspondent à son personnage. Le jeu symbolique mature suppose également, pour des enfants de cet âge, la création de scénarios riches qui intègrent différents éléments familiers (p. ex. famille, transport), les amenant à puiser dans leur mémoire.
Ces différentes informations dévoilent que le jeu symbolique représente un contexte d'observation privilégié à l'éducation préscolaire. En effet, l'observation du jeu permet à l'adulte d'examiner le niveau d'habiletés des enfants pour ensuite les soutenir efficacement dans leur développement et leurs apprentissages, par exemple à l'aide de l'étayage. Concept provenant des travaux de Bruner (1983), l'étayage consiste à accompagner l'enfant pour l'amener de plus en plus loin dans son développement, en tenant compte de ses capacités actuelles et potentielles (Duval et Bouchard, 2013). Dans le cadre du jeu symbolique, cette forme d'accompagnement vise à guider l'enfant afin qu'il parvienne à un jeu de plus en plus mature, pour qu'il puisse développer ses habiletés associées aux FE de manière plus poussée (Bodrova et Leong, 2010). L'étayage permet ainsi à l'adulte de créer des conditions optimales pour la complexification du jeu. Par exemple, l'enseignant pourrait accompagner l'enfant en lui offrant suffisamment de temps pour développer un scénario de jeu de plus en plus évolué, il pourrait rendre accessible du matériel riche et diversifié, il pourrait prendre lui-même un rôle pour suggérer des nouvelles actions aux enfants, etc. (Trawick-Smith, 2012).

Pour que le jeu symbolique devienne une situation d'apprentissage et de développement riche et signifiante, les temps de jeu doivent être suffisamment longs et les enfants doivent pouvoir les planifier, afin de s'engager dans des rôles et des règles implicites (Landry, 2014). Hanline, Milton et Phelps (2010) ajoutent que la fréquence et la complexité du jeu symbolique sont des aspects importants à considérer dans le milieu éducatif, afin que l'enfant développe ses FE de manière efficace. Par exemple, le jeu devrait favoriser le dialogue, en plus d'intégrer l'utilisation d'accessoires symboliques (p. ex. un bloc de bois représentant un téléphone).

Enfin, selon l'approche vygotskienne, le rôle de l'adulte ne consiste pas à diriger le jeu de l'enfant, mais bien à le soutenir afin qu'il se complexifie et qu'il devienne mature. Les pratiques enseignantes déployées dans le cadre du jeu symbolique devraient permettre de bonifier la situation d'apprentissage et de développement dans laquelle l'enfant s'investit, l'amenant alors à développer ses habiletés cognitives liées aux FE.

\subsection{Optimiser le potentiel des stratégies d'observation et intervention}

Bien qu'il ait été démontré que la mise en place de différentes stratégies d'intervention permettrait de soutenir le développement des FE (Bodrova, 2008; Diamond et Lee, 2011), certaines conditions seraient essentielles afin d'optimiser ces dernières et d'en assurer l'efficacité (Bierman et Torres, 2016). Déjà en 1966, Luria soutenait que les fonctions psychologiques supérieures (c.-à-d. FE) sont d'abord développées grâce aux interactions entre l'enfant et les adultes qui l'entourent. Ces interactions permettent notamment à l'enfant de développer son langage, ce qui 
l'amène à modifier ses fonctions mentales, en plus de créer de nouvelles formes d'activité dans son esprit (Luria, 1966).

Selon Bodrova et Leong (2010), il importe de miser sur les temps de jeu permettant des interactions de qualité en classe (adulte-enfant et enfant-enfant), lesquelles favorisent le développement des FE. En ce sens, Moreno et al. (2016) ont récemment montré que les enfants faisaient davantage preuve d'habiletés liées aux FE (p. ex. mémoire de travail, inhibition) lorsque l'adulte interagissait avec eux, voire lorsqu'il les accompagnait dans le cadre de leurs jeux (p. ex. en prenant les traits d'un personnage, en les questionnant sur leurs stratégies, etc.).

De même, les enseignants qui s'engagent dans une activité quotidienne d'observation du jeu deviennent en mesure d'intervenir et d'étayer les situations d'apprentissages et de développement centrées sur l'enfant, et ce, de manière différenciée. Ils peuvent ainsi augmenter ou diminuer le soutien pédagogique offert aux enfants, selon leur rythme unique de développement et d'apprentissage. Cette manière d'intervenir permet à l'enfant de réfléchir à ses actions, en lui procurant un sentiment d'autocontrôle. Dans ce cadre, les activités proposées à l'enfant sont spécifiquement conçues pour inciter l'utilisation d'habiletés liées aux FE.

Plusieurs autres résultats de recherche (p. ex. Bernier et al., 2011; Duval et al., 2016; Fleer et al., 2017) suggèrent que par l'adoption de certaines attitudes (p. ex. sensibilité), l'adulte pourrait mettre en place un environnement propice à l'émergence des FE. En ce sens, il est d'abord suggéré de miser sur la qualité du soutien émotionnel dans le milieu éducatif (Curby et al., 2009). En effet, il semble que les enseignants qui interagissent de manière positive avec les enfants (p. ex. qui instaurent un climat positif, qui démontrent de la sensibilité à l'égard des enfants) sont ensuite en mesure de fournir un soutien de qualité dans leurs apprentissages. Duval et al. (2016) ont d'ailleurs montré que la qualité du soutien émotionnel offert en classe d'éducation préscolaire influençait les habiletés liées à la mémoire de travail et à l'inhibition chez l'enfant âgé de 5 ans.

De plus, les adultes qui organisent la classe en se souciant des besoins et des intérêts de l'enfant (p. ex. en variant les modalités d'apprentissage, en offrant du matériel riche et diversifié) lui permettent de s'investir dans des situations adaptées à son niveau de développement (Curby et al., 2009), pendant lesquelles il pourra mettre en place des habiletés liées aux FE. La structuration de l'horaire (p. ex. routines claires et prévisibles), de même que l'encadrement et la planification des situations proposées en classe inciteraient également l'enfant à explorer son environnement (Ibid.), ce qui favoriserait le développement de ses FE.

Enfin, la disponibilité, l'engagement et le support de l'enseignant seraient associés au niveau de persévérance de l'enfant dans les situations d'apprentissage et de développement suggérées. De manière plus précise, le soutien offert par l'adulte dans les situations d'apprentissage et de développement (p. ex. questionnement, étayage) amène l'enfant à réfléchir et à développer de nouveaux concepts à l'aide de rétroactions constructives et spécifiques (Curby et al., 2009), ce qui favorise en retour le développement des FE. La qualité des rétroactions consiste à se concentrer sur l'évolution de l'apprentissage et de la compréhension de l'enfant, au lieu de simplement indiquer si une réponse est correcte ou non (Franke, Kazemi et Battey, 2007). Les encouragements de l'enseignant permettent ainsi de soutenir le niveau d'engagement de l'enfant dans les situations d'apprentissage et de développement offertes, lequel serait lié au développement des $\mathrm{FE}$, favorisant par le fait même sa réussite éducative (Raver et al., 2008; Raver et al., 2011).

\section{Conclusion}

Cet article a d'abord permis de dégager les perspectives théoriques actuelles qui sont proposées afin de comprendre le développement des FE chez les enfants d'âge préscolaire. Rappelons que les conceptualisations des $\mathrm{FE}$ tendent à se situer selon deux grandes approches : 1) la perspective unitaire, mettant en évidence des composantes communes et indissociables des $\mathrm{FE}$; et 2) la perspective liée à des composantes différenciées et interreliées des FE (Knapp et Morton, 2017). Une perspective évolutive a été proposée quant au développement des FE durant la période préscolaire (3-6 ans). En effet, cet article a montré que la structure des FE se modifie progressivement au fil du développement, en raison des changements développementaux observés dans le cortex préfrontal du cerveau, et qu'il devient donc important d'envisager différentes manières de conceptualiser les FE en cours de développement (Ibid.), notamment à l'âge préscolaire.

Considérant ces faits, et soulignant l'intérêt actuel porté envers l'étude des FE, on peut se questionner sur les habiletés associées aux FE qui s'avèrent observables à l'éducation préscolaire, de manière à accompagner efficacement leur développement. Cet article suggère d'abord de mieux comprendre le développement des FE chez l'enfant d'âge préscolaire afin de dégager des pistes d'observation et d'intervention pour le personnel enseignant.

Selon Bierman et Torres (2016), certaines conditions s'avèrent essentielles afin d'optimiser le développement des FE en classe. Par exemple, il est proposé d'offrir des situations d'apprentissage et de développement misant sur les interactions sociales entre les enfants, ceci permettant le développement de différentes stratégies liées aux FE. Pour ce faire, il est reconnu que les pratiques enseignantes centrées sur l'enfant et sur l'apprentissage par le jeu (p. ex. jeu symbolique mature) lui permettent d'apprendre et de se développer à son plein potentiel pendant la période préscolaire (Bodrova et Leong, 2010 ; Diamond, 2009; Fleer et al., 2017; Moreno et al., 2016). 


\section{Références}

Anderson, V., Jacobs, R. et Anderson, P. (dir.). (2008). Executive functions and the frontal lobes: A lifespan perspective. New York, NY: Taylor \& Francis. https://doi.org/10.4324/9780203837863

Bear, M. F., Connors, B. W. et Paradiso, M. (2016). Neurosciences : À la découverte du cerveau (4 éd.). Paris, France: Pradel.

Bernier, A., Carlson, S. M., Deschênes, M. et Matte-Gagné, C. (2011). Social factors in the development of early executive functioning: a closer look at the caregiving environment. Developmental Science, 15(1), 12-24. https://doi.org/10.1111/j.1467-7687.2011.01093.x

Best, J. R. et Miller, P. H. (2010). A developmental perspective on executive function. Child Development, 81(6), 16411660. https://doi.org/10.1111/j.1467-8624.2010.01499.x

Best, J. R., Miller, P. H. et Jones, L. L. (2009). Executive functions after age 5: Changes and correlates. Developmental Review, 29(3), 180-200. https://doi.org/10.1016/j.dr.2009.05.002

Bierman, K. L. et Torres, M. (2016). Promoting the development of executive functions through early education and prevention programs. Dans J. A. Griffin, P. McCardle et L. S. Freund (dir.), Executive function in preschool-age children: Integrating measurement, neurodevelopment, and translational research (p. 299326). Washington, DC: American Psychological Association. https://doi.org/10.1037/14797-014

Blair, C. B. et Raver, C. C. (2014). Closing the achievement gap through modification of neurocognitive and neuroendocrine function: Results from a cluster randomized controlled trial of an innovative approach to the education of children in kindergarten. PLoS ONE, 9(11), e112393. https://doi.org/10.1371/journal.pone.0112393

Blair, C. B. et Razza, R. P. (2007). Relating effortful control, executive function, and false belief understanding to emerging math and literacy ability in kindergarten. Child Development, 78(2), 647-663.

https://doi.org/10.1111/j.1467-8624.2007.01019.x

Bodrova, E. (2008). Make-believe play versus academic skills: A Vygotskian approach to dilemma of early childhood education. European Early Childhood Education Research Journal, 16(3), 357-369. https://doi.org/10.1080/13502930802291777

Bodrova, E., Germeroth, C. et Leong, D. J. (2013). Play and selfregulation: Lessons from Vygotsky. American Journal of Play, 6(1), 111-123.

http://www.journalofplay.org/issues/6/1/article/7play-and-self-regulation-lessons-vygotsky
Bodrova, E. et Leong, D. J. (2010). Revisiting vygotskian perspectives of play and pedagogy. Dans S. Rogers (dir.), Rethinking play and pedagogy in early childhood education. Concepts, contexts and cultures (p. 60-72). New York, NY: Routledge. http://doi.org/10.4324/9780203839478

Bodrova, E. et Leong, D. J. (2007). Tools of the mind: The Vygotskian approach to early childhood education ( $2^{\mathrm{e}}$ éd.). Columbus, $\mathrm{OH}$ : Merrill/Prentice Hall.

Bouchard, C. (2012). Le développement global de l'enfant, au cœur de l'éducation au préscolaire! Revue Préscolaire, 50(2), 9-14.

https://www.aepq.ca/wpcontent/uploads/2011/04/AEPQ_Revue_v50n2.pdf

Bouchard, C. et Fréchette, N. (2008). Le développement global de l'enfant de 0 à 5 ans en contextes éducatifs. Québec, Canada: Presses de l'Université du Québec.

Bransford, J. D., Brown, A. L. et Cocking, R. R. (2000). How people learn. Washington, DC: National Academy Press. https://doi.org/10.17226/9853

Bruner, J. (1983). Child's talk: Learning to use language. New York, NY: Norton.

Bull, R., Espy, K. A. et Wiebe, S. A. (2008). Short-term memory, working memory, and executive functioning in preschoolers: Longitudinal predictors of mathematical achievement at age 7 years. Developmental Neuropsychology, 33(3), 205-228. https://doi.org/10.1080/87565640801982312

Carlson, S. M., Mandell, D. J. et Williams, L. (2004). Executive function and theory of mind: Stability and prediction from ages 2 to 3. Developmental Psychology, 40(6), 11051122. https://doi.org/10.1037/0012-1649.40.6.1105

Carlson, S. M. et Wang, T. S. (2007). Inhibitory control and emotion regulation in preschool children. Cognitive Development, 22(4), 489-510. https://doi.org/10.1016/j.cogdev.2007.08.002

Chevalier, N. (2010). Les fonctions exécutives chez l'enfant: Concepts et développement. Canadian Psychology, 51(3), 149-163. https://doi.org/10.1037/a0020031

Clark, C. A. C., Pritchard, V. E. et Woodward, L. J. (2010). Preschool executive functioning abilities predict early mathematics achievement Developmental Psychology, 46(5), 1176-1191. https://doi.org/10.1037/a0019672

Clark, C. A. C., Sheffield, T. D., Chevalier, N., Nelson, J. M., Wiebe, S. A. et Espy, K. A. (2013). Charting early trajectories of executive control with the shape school. Developmental Psychology, 49(8), 1481-1493. https://doi.org/10.1037/a0030578 
Curby, T. W., LoCasale-Crouch, J., Konold, T. R., Pianta, R. C., Howes, C., Burchinal, ... et Barbarin, O. (2009). The relations of observed pre-K classroom quality profiles to children's achievement and social competence. Early Education and Development, 20(2), 346-372. https://doi.org/10.1080/10409280802581284

Dekker, M. C., Ziermans, T. B. et Swaab, H. (2016). The impact of behavioural executive functioning and intelligence on math abilities in children with intellectual disabilities. Journal of Intellectual Disability Research, 60(11), 10861096. https://doi.org/10.1111/jir.12276

De Luca, C. R. et Leventer, R. J. (2008). Developmental trajectories of executive functions across the lifespan. Dans V. Anderson, R. Jacobs et P. J. Anderson (dir.), Executive functions and the frontal lobes: A lifespan perspective (p. 57-90). New York, NY: Taylor \& Francis. http://doi.org/10.4324/9780203837863

Denckla, M. B. (1996) A theory and model of executive function: A neuropsychological perspective. Dans G. R Lyon et N. A. Krasnegor (dir.), Attention, memory and executive function (p. 263-278). Baltimore: Paul $\mathrm{H}$ Brookes Publishing.

Dennis, M. (2006). Prefrontal cortex: Typical and atypical development. Dans J. Risberg et J. Grafman (dir.), The frontal lobes: Development, function and pathology (p. 128162). New York, NY : Cambridge University Press. https://doi.org/10.1017/cbo9780511545917.007

Diamond, A. (2016). Why improving and assessing executive functions early in life is critical. Dans J. A. Griffin, P. McCardle et L. S. Freund (dir.), Executive function in preschool-age children: Integrating measurement, neurodevelopment, and translational research (p. 11-43). Washington, DC: American Psychological Association. https://doi.org/10.1037/14797-002

Diamond, A. (2013). Executive functions. Annual Review of Psychology, 64(1), 135-168.

https://doi.org/10.1146/annurev-psych-113011-143750

Diamond, A. (2009). Apprendre à apprendre. Les Dossiers de La Recherche, (34), 89-92.

Diamond, A. (2006). The early development of executive functions. Dans E. Bialystok et F. Craik (dir.), Lifespan cognition: Mechanisms of change (p. 70-95). New York, NY: Oxford University Press.

https://doi.org/10.1093/acprof:oso/9780195169539.00 $\underline{3.0006}$

Diamond, A. (2002). Normal development of prefrontal cortex from birth to young adulthood: Cognitive functions, anatomy, and biochemistry. Dans D. T. Stuss et R. T. Knight (dir.), Principles of frontal lobe function (p. 466503). Londres, Royaume-Uni : Oxford University Press. https://doi.org/10.1093/acprof:oso/9780195134971.003.0029
Diamond, A. et Lee, K. (2011). Interventions shown to aid executive function development in children 4 to 12 years old. Science, 333(6045), 959-964.

https://doi.org/10.1126/science.1204529

Duval, S. et Bouchard, C. (2013). Soutenir la préparation à l'école et à la vie des enfants issus de milieux défavorisés et des enfants en difficulté. Document préparé pour le ministère de la Famille. Québec, Canada : Gouvernement du Québec.

https://www.mfa.gouv.qc.ca/fr/publication/documen ts/preparation-ecole.pdf

Duval, S., Bouchard, C., Pagé, P. et Hamel, C. (2016). Quality of classroom interactions in kindergarten and executive functions among five-year-old children. Cogent Education, 3(1), 1-18. https://doi.org/10.1080/2331186x.2016.1207909

Elias, C. L. et Berk, L. E. (2002). Self-regulation in young children: Is there a role for sociodramatic play? Early Childhood Research Quarterly, 17(2), 216-238. https://doi.org/10.1016/S0885-2006(02)00146-1

Elkonin, D. B. (2005). Chapter 1: The Subject of Our Research: The Developed Form of Play. Journal of Russian $\&$ East European Psychology, 43(1), 22-48.

https://www.tandfonline.com/doi/abs/10.1080/10610 $\underline{405.2005 .11059242}$

Engel de Abreu, P. M. J., Abreu, N., Nikaedo, C. C., Puglisi, M. L., Tourinho, C. J., Miranda, M. C., ... et Martin, R. (2014). Executive functioning and reading achievement in school: A study of Brazilian children assessed by their teachers as "poor readers". Frontiers in Psychology, 5, 550. https://doi.org/10.3389/fpsyg.2014.00550

Engelhardt, L. E., Briley, D. A., Mann, F. D., Harden, K. P. et Tucker-Drob, E. M. (2015). Genes unite executive functions in childhood. Psychological Science, 26(8), 11511163. https://doi.org/10.1177/0956797615577209

Fleer, M., Veresov, N. et Walker, S. (2017). Re-conceptualizing executive functions as social activity in children's playworlds. Learning, Culture and Social Interaction, 14, 1-11. https://doi.org/10.1016/j.lcsi.2017.04.003

Fortin, C. et Rousseau, R. (2012). Psychologie cognitive. Une approche de traitement de l'information. Québec, QC: Presses de l'Université du Québec.

Foy, J. G. et Mann, V. A. (2012). Executive function and early reading skills. Reading and Writing, 26(3) 453-472. https://doi.org/10.1007/s11145-012-9376-5

Franke, M. L., Kazemi, E. et Battey, D. (2007). Mathematics teaching and classroom practice. Dans F. K. Lester (dir.), Second handbook of research on mathematics teaching and learning (p. 225-256). Charlotte, NC: Information Age. 
Friedman, N. P. et Miyake, A. (2004). The relations among inhibition and interference control functions: A latentvariable analysis. Journal of Experimental Psychology: General, 133(1), 101-135. https://doi.org/10.1037/0096$\underline{3445.133 .1 .101}$

Fuhs, M. W. et Day, J. D. (2011). Verbal ability and executive functioning development in preschoolers at head start. Developmental Psychology, 47(2), 404-416. https://doi.org/10.1037/a0021065

Fuhs, M. W., Farran, D. C. et Nesbitt, K. T. (2013). Preschool classroom processes as predictors of children's cognitive self-regulation skills development. School Psychology Quarterly, 28(4), 347-359.

https://doi.org/10.1037/spq0000031

Fujisawa, K. K., Todo, N. et Ando, J. (2016). Genetic and environmental influences on the development and stability of executive functions in children of preschool age: A longitudinal study of Japanese twins. Infant and Child Development, 26(3), e1994. https://doi.org/10.1002/icd.1994

Garon, N., Bryson, S. E. et Smith, I. M. (2008). Executive function in preschoolers: A review using an integrative framework. Psychological Bulletin, 134(1), 31-60. https://doi.org/10.1037/0033-2909.134.1.31

Garon, N., Smith, I. M. et Bryson, S. E. (2013). A novel executive function battery for preschoolers: Sensitivity to age differences. Child Neuropsychology, 20(6), 713-736. https://doi.org/10.1080/09297049.2013.857650

Goble, P. et Pianta, R. C. (2017). Teacher-child interactions in free choice and teacher-directed activity settings: Prediction to school readiness. Early Education and Development, 28(8),1035-1051.

https://doi.org/10.1080/10409289.2017.1322449

Hanline, M. F., Milton, S. et Phelps, P. C. (2010). The relationship between preschool block play and reading and maths abilities in early elementary school: A longitudinal study of children with and without disabilities. Early Child Development and Care, 180(8), 1005-1017. https://doi.org/10.1080/03004430802671171

Hirsh-Pasek, K., Golinkoff, R. M., Berk, L. et Singer, D. (2008). A mandate for playful learning in preschool: Presenting the evidence. New York, NY : Oxford University Press. https://doi.org/10.1093/acprof:oso/9780195382716.001 .0001

Holmes-Bernstein, J. et Waber, D. P. (1990) Developmental neuropsychological assessment: The systemic approach. Dans A. A. Boulton, G. B. Baker et M. Hiscock (dir.), Neuromethods (vol. 17, p. 311-371). Totowa, NJ : Humana Press. https://doi.org/10.1385/0896031330
Hoskyn, M. J., Iarocci, G. et Young, A. R. (dir.). (2017). Executive functions in children's everyday lives: A handbook for professionals in applied psychology. New York, NY: Oxford University Press.

https://doi.org/10.1093/acprof:oso/9780199980864.0 01.0001

Hughes, C., Ensor, R., Wilson, A. et Graham, A. (2009). Tracking executive function across the transition to school: A latent variable approach. Developmental Neuropsychology, 35(1), 20-36. https://doi.org/10.1080/87565640903325691

Huizinga, M., Dolan, C. V. et van der Molen, M. W. (2006). Agerelated change in executive function: Developmental trends and a latent variable analysis. Neuropsychologia, 44(11), 2017-2036. https://doi.org/10.1016/j.neuropsychologia.2006.01.010

Isquith, P. K., Gioia, G. A. et Espy K. A. (2004). Executive function in preschool children: Examination through everyday behavior. Developmental Neuropsychology, 26(1), 403-422. https://doi.org/10.1207/s15326942dn2601_3

Jacob, R. et Parkinson, J. (2015). The potential for schoolbased interventions that target executive function to improve academic achievement: A review. Review of Educational Research, 85(4), 512-552. https://doi.org/10.3102/0034654314561338

Johansson, M., Marciszko, C., Brocki, K. et Bohlin, G. (2015). Individual differences in early executive functions: A longitudinal study from 12 to 36 months. Infant and Child Development, 25(6), 533-549. https://doi.org/10.1002/icd.1952

Kagan, J. et Baird, A. (2004). Brain and behavioral development during childhood. Dans M. S. Gazzaniga (dir.), The cognitive neurosciences (p. 93-103). Cambridge, MA: MIT Press.

Kaufman, C. (2010). Executive function in the classroom: Practical strategies for improving performance and enhancing skills for all students. Baltimore, MA: Brookes Publishing.

Kelly. R. et Hammond, S. (2011). The relationship between symbolic play and executive function in young children. Australasian Journal of Early Childhood, 36, 21-27.

Knapp, K. et Morton, J. B. (2017). Executive functioning: A developmental cognitive neuroscience perspective. Dans M. J. Hoskyn, G. Iarocci et A. R. Young (dir.), Executive functions in children's everyday lives: A handbook for professionals in applied psychology (p. 9-20). New York, NY : Oxford University Press.

https://doi.org/10.1093/acprof:oso/9780199980864.0 $\underline{03.0002}$ 
Kroesbergen, E. H., Van Luit, J., Van Lieshout, E., Van Loosbroek, E. et Van de Rijt, B. (2009). Individual differences in early numeracy: The role of executive functions and subitizing. Journal of Psychoeducational Assessment, 27(3), 226-236. https://doi.org/10.1177/0734282908330586

Landry, S. (2014). Favoriser le développement de la pensée sociale par l'étayage du jeu symbolique chez des enfants qui fréquentent la maternelle cinq ans. Thèse de doctorat inédite. Université Laval, Québec, Canada. http://hdl.handle.net/20.500.11794/24789

Lee, K., Bull, R. et Ho, R. M. H. (2013). Developmental changes in executive functioning. Child Development, 84(6), 19331953. https://doi.org/10.1111/cdev.12096

Lehto, J. E., Juujärvi, P., Kooistra, L. et Pulkkinen, L. (2003). Dimensions of executive functioning: Evidence from children. British Journal of Developmental Psychology, 21(1), 59-80. https://doi.org/10.1348/026151003321164627

Lenroot, R. K. et Giedd, J. N. (2006). Brain development in children and adolescents: Insights from anatomical magnetic resonance imaging. Neuroscience $\varepsilon$ Biobehavioral Reviews, 30(6), 718-729.

https://doi.org/10.1016/j.neubiorev.2006.06.001

Luria, A. R. (1966). Higher cortical functions in man. Oxford, Royaume-Uni : Basic Books.

Mann, T. D., Hund, A. M., Hesson-McInnis, M. S. et Roman, Z. J. (2016). Pathways to school readiness: Executive functioning predicts academic and social-emotional aspects of school readiness. Mind, Brain, and Education, 11(1), 21-31. https://doi.org/10.1111/mbe.12134

Miller, M. R., Giesbrecht, G. F., Müller, U., McInerney, R. J. et Kerns, K. A. (2012). A latent variable approach to determining the structure of executive function in preschool children. Journal of Cognition and Development, 13(3), 395-423.

https://doi.org/10.1080/15248372.2011.585478

Ministère de l'Éducation du Québec (MEQ). (2006). Programme de formation de l'école québécoise. Québec, QC: Gouvernement du Québec.

Ministère de la Famille du Québec (MFA). (2007). Accueillir la petite enfance. Québec: QC: Gouvernement du Québec.

Miyake, A. et Friedman, N. P. (2012). The nature and organization of individual differences in executive functions: Four general conclusions. Current Directions in Psychological Science, 21(1), 8-14.

https://doi.org/10.1177/0963721411429458

Miyake, A., Friedman, N. P., Emerson, M. J., Witzki, A. H., Howerter, A. et Wager, T. D. (2000). The unity and diversity of executive functions and their contributions to complex "frontal lobe" tasks: A latent variable analysis. Cognitive Psychology, 41(1), 49-100.

https://doi.org/10.1006/cogp.1999.0734
Monette, S., Bigras, M. et Lafrenière, M.-A. (2015). Structure of executive functions in typically developing kindergarteners. Journal of Experimental Child Psychology, 140, 120-139. https://doi.org/10.1016/j.jecp.2015.07.005

Moreno, A. J., Shwayder, I. et Friedman, I. D. (2016). The function of executive function: Everyday manifestations of regulated thinking in preschool settings. Early Childhood Education Journal, 45(2), 143-153. https://doi.org/10.1007/s10643-016-0777-y

Organisation de coopération et de développement économiques (OCDE) (2007). Comprendre le cerveau: Naissance d'une science de l'apprentissage. Paris, France: Éditions OCDE. https://doi.org/10.1787/9789264029156-fr

Pellegrini, A. D. (2009). The role of play in human development. New York, NY : Oxford University Press. https://doi.org/10.1093/acprof:oso/9780195367324.00 $\underline{1.0001}$

Raver, C. C., Blair, C. B. et Willoughby, M. (2013). Poverty as a predictor of 4-year-olds' executive function: New perspectives on models of differential susceptibility. Developmental Psychology, 49(2), 292-304. https://doi.org/10.1037/a0028343

Raver, C. C., Jones, S. M., Li-Grining, C. P., Metzger, M., Champion, K. M. et Sardin, L. (2008). Improving preschool classroom processes: Preliminary findings from a randomized trial implemented in Head Start settings. Early Childhood Research Quarterly, 23(1), 10-26. https://doi.org/10.1016/j.ecresq.2007.09.001

Raver, C. C., Jones, S. M., Li-Grining, C., Zhai, F., Bub, K. et Pressler, E. (2011). CSRP's impact on low-income preschoolers' preacademic skills: Self-regulation as a mediating mechanism. Child Development, 82(1), 362-378. https://doi.org/10.1111/j.1467-8624.2010.01561.x

Rhoades, B. L., Greenberg, M. T., Lanza, S. T. et Blair, C. B. (2011). Demographic and familial predictors of early executive function development: Contribution of a person-centered perspective. Journal of Experimental Child Psychology, 108(3), 638-662. https://doi.org/10.1016/j.jecp.2010.08.004

Roebers, C. M. (2017). Executive function and metacognition: Towards a unifying framework of cognitive selfregulation. Developmental Review, 45, 31-51. https://doi.org/10.1016/j.dr.2017.04.001

Röthlisberger, M., Neuenschwander, R., Cimeli, P., Michel, E. et Roebers, C. M. (2011). Improving executive functions in 5- and 6-year-olds: Evaluation of a small group intervention in prekindergarten and kindergarten children. Infant and Child Development, 21(4), 411-429. https://doi.org/10.1002/icd.752

Roy, A. (2012). Les fonctions exécutives chez l'enfant: Approche épistémologique et sémiologique clinique. Revue de neuropsychologie, 4(4), 287-297. https://doi.org/10.3917/rne.044.0287 
Shing, Y. L., Lindenberger, U., Diamond, A., Li, S.-C. et Davidson, M. C. (2010). Memory maintenance and inhibitory control differentiate from early childhood to adolescence. Developmental Neuropsychology, 35(6), 679697. https://doi.org/10.1080/87565641.2010.508546

Simard, M., Tremblay, M.-E., Lavoie, A. et Audet, N. (2013). Enquête québécoise sur le développement des enfants à la maternelle 2012. Portrait statistique pour le Québec et ses régions administratives. Québec, Canada: Institut de la statistique du Québec.

http://www.stat.gouv.qc.ca/statistiques/sante/enfant s-ados/developpement-enfants-maternelle-2012.pdf

Skogan, A. H., Egeland, J., Zeiner, P., Øvergaard, K. R., Oerbeck, B., Reichborn-Kjennerud, T. et Aase, H. (2015). Factor structure of the Behavior Rating Inventory of Executive Functions (BRIEF-P) at age three years. Child Neuropsychology, 22(4), 472-492. https://doi.org/10.1080/09297049.2014.992401

Trawick-Smith, J. (2012). Teacher-child play interactions to achieve learning outcomes. Risks and opportunities. Dans R. C. Pianta (dir.), Handbook of early childhood education (p. 259-277). New York, NY : Guilford Press.

Usai, M. C., Viterbori, P., Traverso, L. et De Franchis, V. (2013). Latent structure of executive function in five- and sixyear-old children: A longitudinal study. European Journal of Developmental Psychology, 11(4), 447-462.

https://doi.org/10.1080/17405629.2013.840578

van der Ven, S. H. G., Kroesbergen, E. H., Boom, J. et Leseman, P. P. M. (2012). The structure of executive functions in children: A closer examination of inhibition, shifting, and updating. British Journal of Developmental Psychology, 31(1), 70-87. https://doi.org/10.1111/j.2044-835X.2012.02079.x

Vocate, D. R. (1987). The theory of A.R. Luria: Functions of spoken language in the development of higher mental processes. Hillsdale, MI: Erlbaum.

Vygotski, L. S. (1997). Pensée et langage (3e éd.). Paris, France: La Dispute.
Vygotsky, L. S. (1978). The role of play in development. Dans L. S. Vygotsky (dir.), Mind in society. The development of higher psychological processes (p. 92-104). Cambridge, MA : Harvard University Press.

Wiebe, S. A., Espy, K. A. et Charak, D. (2008). Using confirmatory factor analysis to understand executive control in preschool children: I. Latent structure. Developmental Psychology, 44(2), 575-587. https://doi.org/10.1037/0012-1649.44.2.575

Wiebe, S. A., Sheffield, T., Nelson, J. M., Clark, C. A. C., Chevalier, N. et Espy, K. A. (2011). The structure of executive function in 3-year-olds. Journal of Experimental Child Psychology, 108(3), 436-452. https://doi.org/10.1016/j.jecp.2010.08.008

Williford, A. P., Vick Whittaker, J. E., Vitiello, V. E. et Downer, J. T. (2013). Children's engagement within the preschool classroom and their development of self-regulation. Early Education \& Development, 24(2), 162-187. http://dx.doi.org/10.1080/10409289.2011.628270

Willoughby, M. T., Blair, C. B., Wirth, R. J. et Greenberg, M. (2010). The measurement of executive function at age 3 years: Psychometric properties and criterion validity of a new battery of tasks. Psychological Assessment, 22(2), 306-317. https://doi.org/10.1037/a0018708

Willoughby, M. T., Wirth, R. J. et Blair, C. B. (2012). Executive function in early childhood: Longitudinal measurement invariance and developmental change. Psychological Assessment, 24(2), 418-431. https://doi.org/10.1037/a0025779

Zelazo, P. D. (2013). The Oxford handbook of developmental psychology (vol.1). New York, NY : Oxford University Press. https://doi.org/10.1093/oxfordhb/9780199958450.001.0001

Zelazo, P. D. et Müller, U.(2010). Executive function in typical and atypical development. Dans U. Goswami (dir.), The WileyBlackwell handbook of childhood cognitive development (p. 574-603). Cambridge, Royaume-Uni : Wiley-Blackwell. https://doi.org/10.1002/9781444325485.ch22 